\title{
Voltammetric Determination of Tinidazole Using a Glassy Carbon Electrode Modified with Single-Wall Carbon Nanotubes
}

\author{
Chunhai YANG \\ Department of Chemistry, Hubei Institute for Nationalities, Enshi 445000, Hubei, P. R. China
}

\begin{abstract}
An electrochemical method based on a single-wall carbon nanotubes (SWNTs) film-coated glassy carbon electrode (GCE) was described for the determination of tinidazole. In a $0.1 \mathrm{M}$ Britton-Robinson buffer with a $\mathrm{pH}$ of 10.0 , tinidazole yields a very sensitive and well-defined reduction peak at $-0.78 \mathrm{~V}$ ( $v s$. SCE) on a SWNTs-modified GCE. Compared with that on a bare GCE, the reduction peak of tinidazole increases significantly on the modified GCE. Thus, all of the experimental parameters were optimized and a sensitive voltammetric method is proposed for tinidazole determination. It is found that the reduction peak current is proportional to the concentration of tinidazole over the range from $5 \times 10^{-8}$ to $4 \times 10^{-5} \mathrm{M}$, and that the detection limit is $1 \times 10^{-8} \mathrm{M}$ at $3 \mathrm{~min}$ open-circuit accumulation. This new analysis method was demonstrated with tinidazole drugs.
\end{abstract}

(Received October 6, 2003; Accepted December 4, 2003)

\section{Introduction}

Carbon nanotubes (CNTs) are molecular-scale wires with high electrical conductivity, extremely high mechanical strength and modulus, and can be divided into two categories: single-wall carbon nanotubes (SWNTs) and multi-wall carbon nanotubes (MWNTs). The so-called SWNTs are made of one layer of graphene sheet, while the MWNTs consist of more than one layer. Since their discovery by Iijima, ${ }^{1} \mathrm{CNTs}$ have attracted much attention in the past decade due to their fascinating and unique structural, mechanical, electrical, and electromechanical properties. It is very interesting and full of challenge to fabricate CNTs-modified electrodes for electrochemical researchers, since the subtle electronic properties suggest that CNTs have the ability to promote electron transfer. However, a major barrier for this is the insolubility of CNTs in all solvents. ${ }^{2}$ Many efforts have been made to disperse CNTs in different solvents, such as DMF, ${ }^{3}$ acetone,${ }^{4}$ concentrated sulfuric acid. ${ }^{5}$

In this work, SWNTs were easily dispersed into water in the presence of a hydrophobic surfactant, and a SWNTs-film was achieved on a surface of the glassy carbon electrode (GCE) via solvent evaporation. This new SWNTs-film was characterized by different methods, and it was found that this cast film-coated electrode could significantly enhance the sensitivity of determining tinidazole.

Tinidazole is an anti-parasitic drug widely used throughout Europe and the Third World as a treatment for a variety of amebic and parasitic infections. A large body of clinical data exists to support its use as a treatment for amebas, giardia and vaginal trichomona. Thus, the determination of tinidazole is of great importance and various methods have been developed. Spectrophotometric ${ }^{6-8}$ and chromatographic methods $\mathrm{s}^{9,10}$ were commonly used to detect tinidazole. Although these spectrophotometric methods require simple equipment, their sensitivity (about $10^{-6} \mathrm{~mol} / \mathrm{L}$ ) is poor. Although

E-mail: papersubmission@hotmail.com chromatographic methods possess higher sensitivity compared with spectrophotometric methods, they require expensive instruments and a much longer detection time. Due to a reduction of the nitro group in the tinidazole molecule, an electrochemical method has also been developed. ${ }^{11}$ However, to the best of our knowledge, the determination of tinidazole using a single-wall carbon nanotubes (SWNTs) modified electrode has not been reported. In the current work, tinidazole yielded a well-defined and very sensitive reduction peak at the SWNTsfilm modified GCE. Compared with that at the bare GCE, the reduction peak current increased remarkably. Based on this, an electrochemical method is proposed for the direct determination of tinidazole. Compared with other methods developed for tinidazole determination, this new procedure possesses following advantages, such as a low detection limit, a rapid response, excellent reproducibility, simplicity and low cost.

\section{Experimental}

\section{Reagents}

All reagents were of analytical-reagent grade, unless otherwise stated. Tinidazole (Sigma, USA), and dihexadecyl hydrogen phosphate (DHP) (Fluka) were used without purification. Tinidazole stock solutions under voltammetric investigation were renewed daily and prepared in a Britton-Robinson buffer. Double-distilled water was used throughout.

Single-wall carbon nanotubes (SWNTs) were prepared by a direct-current arc-discharge method ${ }^{12}$ and purified to $>90 \% .^{13}$ They were then refluxed for $10 \mathrm{~h}$ in concentrated $\mathrm{HNO}_{3}$ to cause segmentation and carboxylation. ${ }^{14}$

\section{Apparatus}

A CHI 660A (CH Instrument, Austin, USA) was used for electrochemical measurements, and a conventional threeelectrode system, including a SWNTs-film modified GC working electrode, a saturated calomel reference electrode 


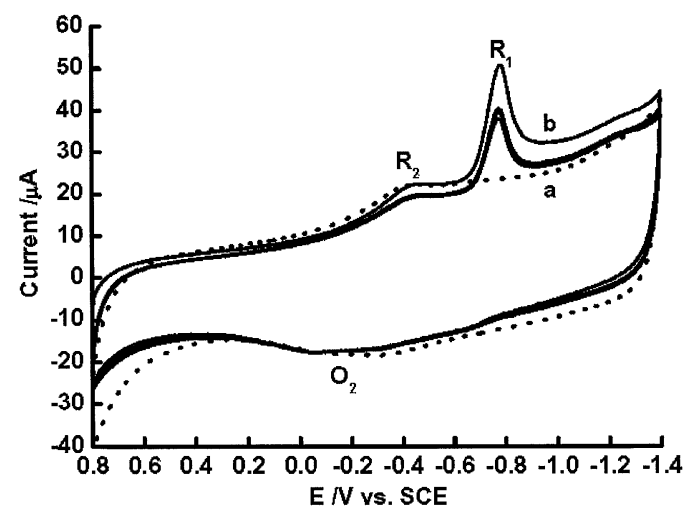

Fig. 1 Cyclic voltammograms of a SWNTs-modified GCE. Curve (a), in a $0.1 \mathrm{M}$ Britton-Robinson buffer with a $\mathrm{pH}$ of 10.0; curve (b), (a) $+5 \times 10^{-5} \mathrm{~mol} / \mathrm{L}$ tinidazole. Scan rate, $100 \mathrm{mV} / \mathrm{s}$.

(SCE) and a Pt wire counter electrode, was employed.

\section{Surface preparation and modification}

After 2.5-mg SWNTs and 5-mg DHP were dispersed into 5 $\mathrm{mL}$ of redistilled water by ultrasonication agitation for about 20 min, a homogeneous and stable black suspension was obtained. Prior to modification, the GCE was mechanically polished with alumina paste of different grades to a mirror finish, rinsed and sonicated ( $3 \mathrm{~min})$ in redistilled water. Finally, the GCE was coated with $10 \mu \mathrm{L}$ of the SWNTs-DHP suspension and allowed to evaporate water at room temperature in the air. The DHPfilm coated GCE was prepared by the same procedure as explained above, but without SWNTs.

\section{Analytical procedure}

After $10 \mathrm{~mL} \mathrm{pH} 10.0$ Britton-Robinson buffer $(0.1 \mathrm{~mol} / \mathrm{L})$ was placed in the electrochemical cell, the required volume of tinidazole standard solutions was added by a micropipette. The solution was deaerated with nitrogen for $10 \mathrm{~min}$, and accumulation was carried out under an open-circuit for $3 \mathrm{~min}$. After that, differential pulse voltammograms (DPVs) from -0.40 to $-1.00 \mathrm{~V}$ were recorded after a $15 \mathrm{~s}$ quiet time. The reduction-peak current was measured at $-0.78 \mathrm{~V}$. Prior to and after every measurement, the SWNTs-film coated GCE was activated by five successive cyclic voltammetric sweeps between 0.0 to $-1.20 \mathrm{~V}$ at $100 \mathrm{mV} / \mathrm{s}$ in a $\mathrm{pH} 10.0$ Britton-Robinson buffer to produce a reproducible electrode surface.

\section{Results and Discussion}

\section{Electrochemical behaviors of tinidazole}

The electrochemical behaviors of tinidazole at a SWNTs-film coated GCE have been investigated using cyclic voltammetry (CV). Figure 1a shows cyclic voltammograms of the SWNTsfilm coated GCE in a $\mathrm{pH} 10.0$ Britton-Robinson buffer $(0.1$ $\mathrm{mol} / \mathrm{L}$ ). A couple of redox peaks with potentials of $-0.30 \mathrm{~V}$ and $-0.42 \mathrm{~V}$ can be observed, which is similar to that of other carbon nanotubes modified electrodes. ${ }^{3}$ Upon the addition of 5 $\times 10^{-5} \mathrm{~mol} / \mathrm{L}$ tinidazole, a sensitive and well-defined reduction peak appears at $-0.78 \mathrm{~V}$ during the first cathodic sweep from $0.80 \mathrm{~V}$ to $-1.40 \mathrm{~V}$ (Fig. 1b). Upon a reverse scan, no corresponding oxidation peak is observed, revealing that the electrode reaction of tinidazole is totally irreversible. According to the currently accepted mechanism for the

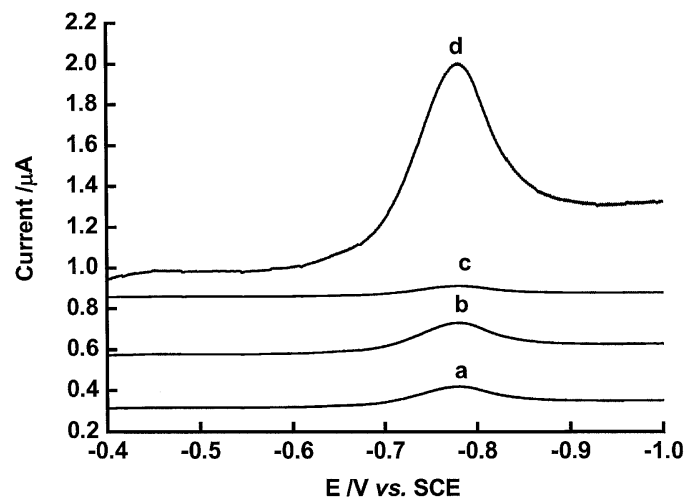

Fig. 2 DP voltammograms of $5 \times 10^{-7} \mathrm{~mol} / \mathrm{L}$ tinidazole at three different electrodes in a pH 10.0 Britton-Robinson buffer. Curve (a), bare GCE; curve (b), bare graphite electrode; curve (c), DHPmodified GCE; curve (d), SWNTs-film coated GCE. Accumulation time, $3 \mathrm{~min}$; pulse amplitude, $50 \mathrm{mV}$; scan rate, $20 \mathrm{mV} / \mathrm{s}$; pulse width, $50 \mathrm{~ms}$.

electroreduction of aromatic and heteroaromatic nitro compounds, the reduction peak is attributed to a four-electron reduction of the nitro group to the corresponding hydroxylamine, ${ }^{15,16}$ and may be written as follows:

The reduction-peak current in the second cyclic sweep decreases remarkably compared with that of the first cyclic sweep. After the second cyclic sweep, the peak current decreases slightly and finally almost remains unchangeable. This phenomenon may be caused by the fact that the adsorption of tinidazole, or its reductive product, occurs at the electrode surface, and hence inactivates the electrode surface.

$$
\mathrm{R}-\mathrm{NO}_{2}+4 \mathrm{e}^{-}+4 \mathrm{H}^{+} \longrightarrow \mathrm{R}-\mathrm{NHOH}+\mathrm{H}_{2} \mathrm{O}
$$

The voltammetric response of $5 \times 10^{-7} \mathrm{~mol} / \mathrm{L}$ tinidazole at four different electrodes was compared by differential pulse voltammetry (DPV). In a pH 10.0 Britton-Robinson buffer and after $3 \mathrm{~min}$ of open-circuit accumulation, a low reduction peak appeared at $-0.78 \mathrm{~V}$ at the bare GCE (Fig. 2a). Under identical conditions, the reduction peak current increased very slightly at a bare graphite electrode (Fig. 2b); however, it decreased at the DHP-film coated GCE (Fig. 2c). The peak current decrease may have been caused by the fact that the DHP forms a perfect film on the GCE surface, and thus inhibits electron transfer. Compared with that at both bare GCE and a graphite electrode, the reduction peak for the SWNTs-film coated GCE increases significantly under comparable conditions, and the reduction peak potential does not change (Fig. 2d). The remarkable peak current enhancement is undoubtedly attributed to the extraordinary properties of SWNTs, such as subtle electronic properties, high aspect ration and strong adsorptive ability.

\section{Effects of supporting electrolytes}

The electrochemical properties of $5 \times 10^{-7} \mathrm{~mol} / \mathrm{L}$ tinidazole in various medium, such as pH $5.0-8.0$ phosphate buffer, $\mathrm{pH} 2.0$ 8.0 Macllvaine buffer, and $\mathrm{pH}$ 2.0-11.0 Britton-Robinson buffer (each $0.1 \mathrm{~mol} / \mathrm{L}$ ), were investigated by DPV. It was found that the peak current is highest and the peak shape is well-defined in $\mathrm{pH} 10.0$ Britton-Robinson buffer. 


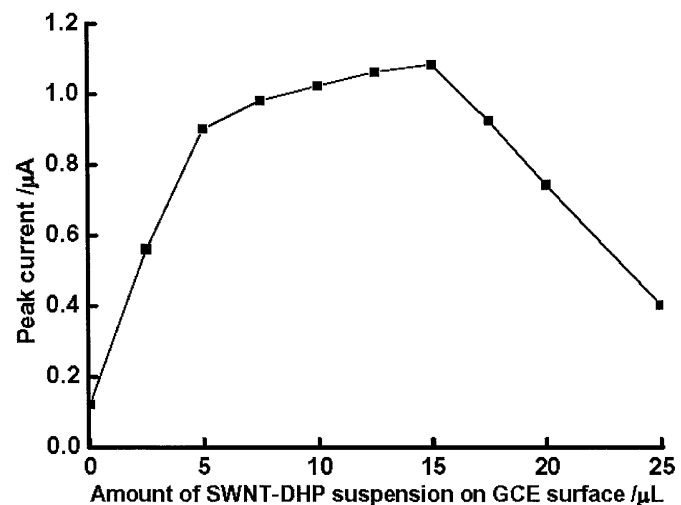

Fig. 3 Influences of the amount of SWNTs-DHP suspension on the reduction peak current of $5 \times 10^{-7} \mathrm{~mol} / \mathrm{L}$ tinidazole. All other conditions are the same as in Fig. 3.

In a $0.1 \mathrm{~mol} / \mathrm{L}$ Britton-Robinson buffer, the influences of the $\mathrm{pH}$ on the reduction peak potential were examined by linear sweep voltammetry (LSV). The reduction peak potential $\left(E_{\mathrm{pc}}\right)$ shifts positively as the $\mathrm{pH}$ decreases from 11.0 to 3.0, and obeys the following equations: $E_{\mathrm{pc}}=-0.21-0.057 \mathrm{pH}(r=0.997)$. The slope of $57 \mathrm{mV} / \mathrm{pH}$ indicates that equal number of electrons and protons are involved in the reduction of tinidazole.

\section{Influences of the SWNTs-DHP film thickness}

Generally, the thickness of the SWNTs-DHP cast film, which is determined by the amount of the SWNTs-DHP suspension on the GCE surface, has an obvious effect on the current responses of tinidazole. Figure 3 depicts the reduction-peak current responses for various amounts of the SWNTs-DHP suspension. The reduction peak current gradually increases while gradually increasing the volume of the SWNTs-DHP suspension from 0 to $10 \mu \mathrm{L}$. When the amount of SWNTs-DHP suspension increases from 10 to $15 \mu \mathrm{L}$, the peak current changes slightly. However, when it exceeds $15 \mu \mathrm{L}$, the peak current conversely decreases. SWNTs are ideal electrode materials with excellent electrical conductivity. In principle, the reduction peak current is almost independent of the thickness of the SWNTs cast film. However, DHP is an insulator, and can lower the electrical conductivity of the cast film, and finally lowers the electron-transfer rate. Hence, the peak current contrarily decreases when the SWNTsDHP film is too thick.

\section{Optimization of accumulation conditions}

The reduction peak current of $5 \times 10^{-7} \mathrm{~mol} / \mathrm{L}$ tinidazole was compared after 3 min of accumulation under different potential by DPV. The peak current almost remained unchangeable as the accumulation potential shifted from 0.50 to $-0.60 \mathrm{~V}$, revealing that the accumulation potential had no influence on the reduction peak current of tinidazole at the SWNTS-film coated GCE. Thus, an open-circuit accumulation was employed.

The influences of the accumulation time on the reduction peak current of $5 \times 10^{-7} \mathrm{~mol} / \mathrm{L}$ tinidazole have been examined; the results are illustrated in Fig. 4. The reduction peak current increases greatly within the first $3 \mathrm{~min}$ and then levels off, suggesting that the accumulation of tinidazole is very rapid to reach saturation at the SWNTs-film.

\section{Calibration graph}

The calibration curve for tinidazole in $\mathrm{pH} \quad 10.0$

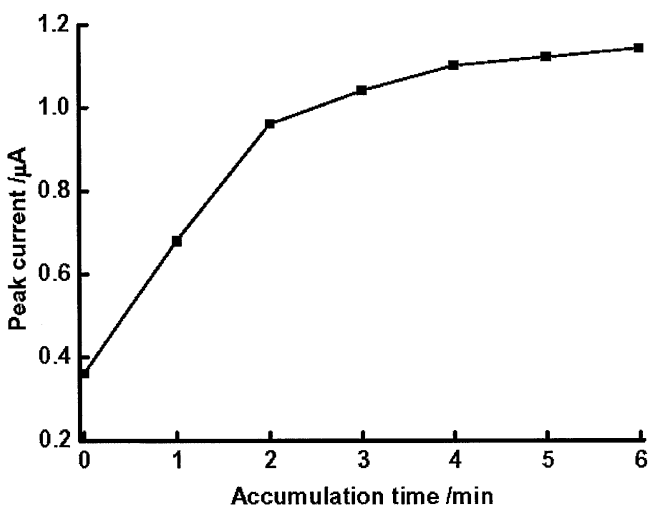

Fig. 4 Effects of the accumulation time on the reduction peak current of $5 \times 10^{-7} \mathrm{~mol} / \mathrm{L}$ tinidazole. Other conditions are the same as in Fig. 3.

Britton-Robinson buffer was measured by DPV. The best parameters on the SWNTs-film coated GCE are an accumulation time of $3 \mathrm{~min}$, a pulse amplitude of $50 \mathrm{mV}$, a scan rate of $20 \mathrm{mV} / \mathrm{s}$, and a pulse width of $50 \mathrm{~ms}$. The linear segment increases from $5 \times 10^{-8}$ to $4 \times 10^{-5} \mathrm{~mol} / \mathrm{L}(r=0.998)$ with a slope of $1.01 \times 10^{7} \mu \mathrm{A}(\mathrm{mol} / \mathrm{L})^{-1}$ and an intercept of 0.03 $\mu \mathrm{A}$. The detection limit of $1 \times 10^{-8} \mathrm{~mol} / \mathrm{L}$ was obtained at 3 min of accumulation. The relative standard deviation (RSD) of $5.8 \%$ for $5 \times 10^{-7} \mathrm{~mol} / \mathrm{L}$ tinidazole $(n=10)$ showed excellent reproducibility.

The long-term stability of the SWNTs-film coated GCE was evaluated by measuring the current responses at a fixed tinidazole concentration of $5 \times 10^{-7} \mathrm{~mol} / \mathrm{L}$ over a period of 4 weeks. The SWNTs-film coated GCE was used daily and stored in the air. The experimental results indicated that the current responses deviated by only $7.2 \%$, suggesting that the SWNTs-film coated GCE possesses long-term stability.

\section{Interferences}

To evaluate the interferences of foreign compounds on the determination of tinidazole at the $5 \times 10^{-7} \mathrm{~mol} / \mathrm{L}$ level, a systematic study was carried out. The results are given in Table 1. It is found that a 500-fold concentration of uric acid (UA), dopamine (DA), ascorbic acid (AA), xanthine (XA), vitamine E, vitamine $\mathrm{A}$, progesterone, caffeine and cholesterol almost do not influence the current response of $5 \times 10^{-7} \mathrm{~mol} / \mathrm{L}$ tinidazole (signal change below 5\%). However, some compounds containing a nitro group, such as metronidazole and nitrophenols, interfere with the determination of tinidazole, because they contain the same reductive groups that can be reduced near the potentials of tinidazole.

\section{Determination of tinidazole in drugs}

The SWNTs-film coated GCE was used to detect tinidazole in tablets and injections. No pretreatment for the tinidazole injection was made, except for dilution with the Britton-Robinson buffer ( $\mathrm{pH}$ 10.0).

After the average mass of five tablets was determined and finely powdered, the required amount of sample used to prepare a solution of $c a \cdot 10^{-3} \mathrm{~mol} / \mathrm{L}$ was transferred into a $100-\mathrm{mL}$ standard flask containing $80 \mathrm{~mL}$ of Britton-Robinson buffer $(\mathrm{pH}$ 10.0). The contents of the flask were stirred magnetically for 15 min, and then diluted to volume with the same supporting electrolyte. The solution was filtered and the first $20 \mathrm{~mL}$ of the filtrate was removed. Appropriate solutions were prepared by 
Table 1 Interferences of other compounds on the reduction peak current of $5 \times 10^{-7} \mathrm{~mol} / \mathrm{L}$ tinidazole

\begin{tabular}{lcc}
\hline \multicolumn{1}{c}{ Interferent } & Concentration/mol L & Signal change, $\%$ \\
\hline Uric acid (UA) & $2.5 \times 10^{-4}$ & +2.64 \\
Dopamine (DA) & $2.5 \times 10^{-4}$ & -3.46 \\
Ascorbic acid (AA) & $2.5 \times 10^{-4}$ & +1.38 \\
Xanthine (XA) & $2.5 \times 10^{-4}$ & -2.04 \\
Vitamine E & $2.5 \times 10^{-4}$ & +1.16 \\
Vitamine A & $2.5 \times 10^{-4}$ & +1.02 \\
Progesterone & $2.5 \times 10^{-4}$ & +0.46 \\
Caffeine & $2.5 \times 10^{-4}$ & -3.68 \\
Cholesterol & $2.5 \times 10^{-4}$ & -3.21 \\
Metronidazole & $2.5 \times 10^{-6}$ & +14.6 \\
4-Nitrophenol & $2.5 \times 10^{-6}$ & +8.64 \\
\hline
\end{tabular}

Table 2 Determination of tinidazole by the SWNTs-film coated GCE and spectrophotometric method

\begin{tabular}{lcccccc}
\hline & \multicolumn{2}{c}{ Proposed voltammetric method } & & \multicolumn{2}{c}{ Spectrophotometry $^{6}$} \\
\cline { 2 - 3 } \cline { 5 - 6 } & $\begin{array}{c}\text { Tablet/ } \\
\text { mg tablet }^{-1}\end{array}$ & $\begin{array}{c}\text { Injection/ } \\
\mathrm{mg} \mathrm{ml}^{-1}\end{array}$ & & $\begin{array}{c}\text { Tablet/ } \\
\text { mg tablet }^{-1}\end{array}$ & $\begin{array}{c}\text { Injection/ } \\
\mathrm{mg} \mathrm{ml}^{-1}\end{array}$ \\
\hline Mean $^{\mathrm{a}}$ & 0.28 & 5.18 & & 0.19 & 5.08 \\
RSD, \% & 4.3 & 3.5 & & 3.2 & 2.9 \\
\hline
\end{tabular}

RSD: relative standard deviation.

a. Mean of five experiments.

taking suitable aliquots of the clear filtrate and diluting them with the supporting electrolyte mentioned above.

Voltammograms were recorded as in standard tinidazole. The content of tinidazole was calculated from the regression equation. The results obtained by SWNTs-film coated GCE were compared with those determined by spectrophotometry. ${ }^{6}$ The results (Table 2) show a good agreement. Further, in order to establish the suitability of the proposed method, known amounts of the standard tinidazole were added into the analytical solution of the pharmaceutical products and the procedure was applied. The recoveries indicate that the accuracy and repeatability of the proposed voltammetric method are very good (Table 3). From the results given in Tables 2 and 3 , it is very clear that this novel SWNTs-modified electrode has great potential for practical sample analysis.

\section{Conclusion}

The carbon nanotubes-modified electrode described in this paper is very cheap and easy to prepare. Due to its unique properties, such as high specific surface area, subtle electronic
Table 3 Recovery studies by the proposed electrochemical method at SWNTs-film coated GCE

\begin{tabular}{lcc}
\hline \multicolumn{1}{c}{ Sample } & Recovery, $\%$ & RSD, $\%$ \\
\hline Tablet (No. 030512) & 103.5 & 4.6 \\
Tablet (No. 030716) & 98.7 & 3.8 \\
Injection (No. 021010604) & 99.2 & 3.4 \\
Injection (No. 030810606) & 102.6 & 3.1 \\
\hline
\end{tabular}

properties and strong adsorptive ability, the SWNTs-film modified GCE shows an obvious electrocatalytic ability for the reduction of tinidazole, since it enhances the peak current significantly. Hence, a very sensitive and simple electrochemical method by using the SWNTs-film coated GCE was developed for the direct determination of tinidazole.

\section{References}

1. S. Iijima, Nature, 1991, 56, 354.

2. J. Wang, M. Musameh, and Y. Lin, J. Am. Chem. Soc., 2003, 125, 2408

3. H. X. Luo, Z. J. Shi, N. Q. Li, Z. N. Gu, and Q. K. Zhuang, Anal. Chem., 2001, 73, 915.

4. F. H. Wu, G. C. Zhao, and X. W. Wei, Electrochem. Commun., 2002, 4, 690.

5. M. Musamech, J. Wang, A. Merkoci, and Y. H. Lin, Electrochem. Commun., 2002, 4, 743.

6. L. Lopez-Martina, F. J. Luna Vazquez, and P. L. Lopez-de-Alba, Anal. Chim. Acta, 1997, 340, 241.

7. P. Nagaraja, K. R. Sunitha, R. A. Vasantha, and H. S. Yathirajan, J. Pharm. Biomed. Anal., 2002, 28, 527.

8. J. P. Salo and H. Sulomies, J. Pharm. Biomed. Anal., 2003, 31, 523

9. J. P. Salo and H. Sulomies, J. Pharm. Biomed. Anal., 1996, $14,1261$.

10. J. P. Salo and H. Sulomies, J. Pharm. Biomed. Anal., 1996, 14, 1267.

11. A. Z. Abu Zuhri, S. Al-Khalil, R. M. Shubietah, and I. ElHroub, J. Pharm. Biomed. Anal., 1999, 21, 881.

12. Z. Shi, Y. Lian, X. Zhou, Z. Gu, Y. Zhang, S. Iijima, L. Zhou, K. T. Yue, and S. Zhang, Carbon, 1999, 37, 1449.

13. Z. Shi, Y. Lian, F. Liao, X. Zhou, Z. Gu, Y. Zhang, and S. Iijima, Solid State Commun., 1999, 112, 35.

14. S. C. Tsang, Y. K. Cheng, P. J. E. Harris, and M. L. H. Green, Nature, 1994, 372, 159.

15. P. Zuman and Z. Fijalek, J. Electroanal. Chem., 1990, 296, 589.

16. A. E. Jammal, J. C. Vire, G. J. Patriarche, and O. N. Palmeiro, Electroanalysis, 1992, 4, 57. 